\title{
Multiscale Finite-Difference-Diffusion-Monte-Carlo Method for Simulating Dendritic Solidification
}

\author{
Mathis Plapp and Alain Karma \\ Physics Department and Center for Interdisciplinary Research on Complex Systems, \\ Northeastern University, Boston, Massachusetts 02115
}

(January 11, 2000)

\begin{abstract}
We present a novel hybrid computational method to simulate accurately dendritic solidification in the low undercooling limit where the dendrite tip radius is one or more orders of magnitude smaller than the characteristic spatial scale of variation of the surrounding thermal or solutal diffusion field. The first key feature of this method is an efficient multiscale diffusion Monte-Carlo (DMC) algorithm which allows off-lattice random walkers to take longer and concomitantly rarer steps with increasing distance away from the solid-liquid interface. As a result, the computational cost of evolving the large scale diffusion field becomes insignificant when compared to that of calculating the interface evolution. The second key feature is that random walks are only permitted outside of a thin liquid layer surrounding the interface. Inside this layer and in the solid, the diffusion equation is solved using a standard finite-difference algorithm that is interfaced with the DMC algorithm using the local conservation law for the diffusing quantity. Here we combine this algorithm with a previously developed phase-field formulation of the interface dynamics and demonstrate that it can accurately simulate three-dimensional dendritic growth in a previously unreachable range of low undercoolings that is of direct experimental relevance.
\end{abstract}

\section{INTRODUCTION}

Diffusion-limited pattern formation, which leads to the spontaneous emergence of complex branched structures, occurs in numerous contexts. A few examples include dendritic solidification [1], electrochemical deposition [2] and corrosion, and the growth of bacterial colonies [3]. Two distinct length scales are typically involved in this class of problems: one that characterizes the pattern itself, such as the thickness of a branch, and one that characterizes the diffusion field associated with the transport of heat or matter. In many cases, these two scales are vastly different. For example, in solidification, the decay length of the thermal or solutal field ahead of a growing dendrite (in a pure or alloy melt) can be one to three orders of magnitude larger than the tip radius of one of its primary branches. Non-trivial pattern formation dynamics can be expected to occur on all intermediate scales. This poses a serious challenge for numerical simulations since a precise integration of the equations of motion on the pattern scale requires a good resolution of the interfacial region, and such a resolution is completely inefficient (i.e. much too fine) to treat the large scale diffusion field. Therefore, in order to retain this precision on the small scale and, at the same time, simulate the pattern evolution on sufficiently large length and time scales, it is necessary to use some form of multiscale algorithm.

Multi-grid and finite element methods with nonuniform meshing represent one possible solution for this type of problems. Their application, however, in the context of growth simulations faces the additional difficulty of a moving interface, which implies that the structure of the simulation grid has to be dynamically adapted. For the classic problem of dendritic crystal growth, sev- eral multi-grid [4 or adaptive meshing algorithms [5] have been proposed in recent years. The most precise to date is the method of Provatas et al. which uses the phase-field model on a regular grid on the scale of the dendrite, whereas the diffusion field is integrated on an adaptive mesh using finite element techniques [6]. While this method appears to be promising, it has yet to be implemented in three dimensions where the difficulty of adaptive meshing becomes significantly enhanced.

We present in this paper an alternative solution to solve this computational challenge and we illustrate its application in the context of the dendritic crystallization of a pure substance from its undercooled melt, even though this algorithm can be applied to any diffusionlimited growth problem for which an explicit solver of the interface dynamics is available. The idea is to use a hybrid approach. The interface dynamics is treated using deterministic equations of motion, in particular those of the phase-field model for the dendritic growth problem considered here. On the other hand, the large-scale diffusion field is represented by an ensemble of off-lattice random walkers and is evolved using a Diffusion Monte Carlo (DMC) algorithm. The two solutions are connected at some distance from the moving interface. The key point for rendering our method efficient is that we use random walkers which dynamically adapt the average length of their random steps. Far from the interface, the walkers can make large jumps and hence be updated only rarely without affecting the quality of the solution near the growing interface. In some sense, our method can be seen as an "adaptive grid algorithm without grid". The DMC algorithm and the connection between deterministic and stochastic parts are rather simple and straightforward to implement in both two and three dimensions, both on 
single-processor and parallel architectures. We demonstrate in this paper that our method is precise, robust, and reliable, and hence constitutes a powerful alternative to state of the art adaptive meshing techniques. Technically, the algorithm bears many similarities to quantum Monte Carlo methods [7]. It is therefore remarkable that the gap between mesoscopic and macroscopic length scales can be bridged using a method borrowed from microscopic physics in an interfacial pattern formation context, which was not a priori obvious to us at the start of this investigation.

Our algorithm builds on ideas of earlier random walk algorithms for simulating pattern formation during viscous fingering [8,9] and solidification [10 13], but introduces two essential new features. Firstly, random walks with variable step size have been used previously in simulations of large-scale diffusion-limited aggregation [14], but only one walker at a time was simulated, and hence the time variable did not explicitly appear in the treatment of the walkers. In the present diffusive case, the memory of the past history, stored in the diffusion field, is essential to the problem. Our DMC algorithm works with a whole ensemble of walkers in "physical" time and hence constitutes a true multiscale solver for the full diffusion problem. Secondly, the algorithms mentioned above use a lattice both to evolve the walkers and to represent the position of the interface by the bonds between occupied (solid) and empty (liquid) sites. Walkers are created or absorbed directly at this interface. The discretization of space and the stochastic creation and absorption of walkers make it difficult to control accurately the interfacial anisotropy and the noise that both play a crucial role in dendritic evolution [11,15]. Consequently, the algorithms aimed at describing dendritic growth [10,11,13], while correctly reproducing all the qualitative features of the growth process, are unable to yield quantitative results that can be tested against experiments. We solve both problems by creating and absorbing walkers not at the solid-liquid interface, but at a "conversion boundary" at some fixed distance from the interface. This means that the stochastic representation of the diffusion and the motion of the interface can be treated separately, which allows us to evolve the interface accurately by the phasefield method using a finite difference representation of controlled precision. At the same time, the stochastic noise created by the DMC algorithm is rapidly damped by the deterministic diffusion in the "buffer layer" between the conversion boundary and the solid-liquid interface, and hence the amplitude of the fluctuations at the solid-liquid interface can be reduced to a prescribed level without much cost in computation time by increasing the thickness of the buffer layer. This is an important issue for simulations of dendritic growth, because the amplification of microscopic fluctuations of the interface is believed to be the main cause for the formation of secondary dendrite branches [16], and it is well known that numerical noise can lead to the formation of spurious sidebranches in simulations. Consequently, we have to demonstrate that the walker noise of our algorithm can be reduced to a level that does not affect the pattern evolution.

Another benefit of the buffer layer is that it makes the algorithm very versatile. Away from the interface, only the standard diffusion equation has to be solved. Therefore, the DMC part of the algorithm and the conversion process between deterministic and stochastic solutions are completely independent from the method used for simulating the interface dynamics, and can easily be carried over to other free boundary problems.

The purpose of the present paper is to describe the algorithm in detail and to demonstrate its reliability and precision by benchmark simulations. Some results concerning three-dimensional crystal growth at low undercoolings have already been presented elsewhere [17, 18, and hence we will focus here on the computational aspects of the problem. Section II contains a brief introduction to dendritic solidification and the basic equations of motion, and describes the phase-field method. In section III, the DMC algorithm and its interfacing with the phase-field equations are described in detail. In section IV, we present results of benchmark simulations, assess the efficiency of the code and the magnitude of numerical noise, and present simulations of three-dimensional dendritic growth. Section V contains a conclusion and the outline of future work.

\section{DENDRITIC GROWTH AND THE PHASE-FIELD METHOD}

When a crystal grows from an undercooled melt, it develops into an intricate branched structure, called a dendrite. This phenomenon has been of central importance to the understanding of spontaneous pattern formation during phase transformations and the emergence of branched structures 19 21]. In addition, it is of considerable practical interest, because dendrites form during the solidification of many commercially important alloys and influence the mechanical properties of the finished material.

We will focus on the dendritic solidification of a pure substance from its homogeneously undercooled melt, starting from a single supercritical nucleus 22 26. This situation is well described by the symmetric model of solidification, which assumes that the diffusivity and thermophysical quantities such as the specific heat and the density are equal for the solid and the liquid phases. During the growth of the crystal, the latent heat of melting is released, and in the absence of convection, the growth becomes limited by the diffusion of heat away from the growing dendrite. The state of the system at any time is described by the temperature field $T(\vec{x}, t)$ and the shape $\Gamma(t)$ of the boundary between solid and liquid. It is customary to define a dimensionless temperature field 


$$
u(\vec{x}, t)=\frac{T(\vec{x}, t)-T_{m}}{L / c_{p}},
$$

where $L$ and $c_{p}$ are the latent heat of melting and the specific heat, respectively, and $T_{m}$ is the melting temperature. In terms of this field, the equations of motion of the symmetric model are

$$
\begin{gathered}
\partial_{t} u=D \vec{\nabla}^{2} u \\
v_{n}=D \hat{n} \cdot\left(\left.\vec{\nabla} u\right|_{S}-\left.\vec{\nabla} u\right|_{L}\right), \\
u_{\Gamma}=-d_{0} \sum_{i=1}^{d-1}\left[a(\hat{n})+\frac{\partial^{2} a(\hat{n})}{\partial \theta_{i}^{2}}\right] \frac{1}{R_{i}}-\beta(\hat{n}) v_{n},
\end{gathered}
$$

where $D$ is the thermal diffusivity, $v_{n}$ is the normal velocity of the interface, and $\hat{n}$ is the unit normal vector of the surface $\Gamma$ pointing towards the liquid. The diffusion equation, Eq. (2) is valid everywhere (in the liquid and in the solid) except on the surface $\Gamma$. The Stefan condition, Eq. (3), valid on $\Gamma(t)$, expresses the conservation of enthalpy at the moving phase boundary. Here, $\left.\vec{\nabla} u\right|_{S}$ and $\left.\vec{\nabla} u\right|_{L}$ denote the limits of the temperature gradient when $\Gamma$ is approached from the solid and the liquid side, respectively, and the equation states that the local heat flux at the interface must be equal to the latent heat generated or consumed during the phase transformation; $v_{n}$ is positive if the solid grows (i.e. freezing). The dimensionless temperature at the interface $u_{\Gamma}$ is given by the generalized Gibbs-Thomson condition Eq. (位). The first term on the right hand side (RHS) is the anisotropic form of the local equilibrium condition (Gibbs-Thomson condition) which relates the temperature to the curvature of the interface and the anisotropic surface tension $\gamma(\hat{n})=\gamma_{0} a(\hat{n})$. For a crystal with cubic symmetry in three dimensions, the anisotropy function $a(\hat{n})$ is usually written as

$$
a(\hat{n})=\left(1-3 \epsilon_{4}\right)\left[1+\frac{4 \epsilon_{4}}{1-3 \epsilon_{4}}\left(n_{x}^{4}+n_{y}^{4}+n_{z}^{4}\right)\right],
$$

where $\epsilon_{4}$ is the anisotropy parameter. Note that in two dimensions $(d=2)$, this expression reduces to

$$
a(\theta)=1+\epsilon_{4} \cos (4 \theta),
$$

where $\theta$ is the angle between the normal and one of the axes of symmetry. On the RHS of Eq. (何),

$$
d_{0}=\frac{\gamma_{0} T_{m} c_{p}}{L^{2}}
$$

is the capillary length, $d$ is the spatial dimension, $\theta_{i}$ are the angles between the normal $\hat{n}$ and the two local principal directions on $\Gamma$, and $R_{i}$ are the principal radii of curvature. Finally, the second term on the RHS of Eq. (1) describes the shift of the interface temperature due to molecular attachment kinetics, and $\beta(\hat{n})$ is the orientation-dependent linear kinetic coefficient. Kinetic effects are believed to be small for the range of solidification speeds of interest here. We will therefore focus on the case where the interface kinetics vanish $(\beta(\hat{n}) \equiv 0)$, which corresponds to local equilibrium at the interface. In this case, the physical length and time scales are set by the capillary length and the diffusivity, and the control parameters of the problem are the anisotropy $\epsilon_{4}$ and the dimensionless undercooling

$$
\Delta=\frac{T_{m}-T_{0}}{L / c_{p}}
$$

where $T_{0}$ is the initial temperature, $T(\vec{x}, 0)=T_{0}$, which provides the thermodynamic driving force for solidification. We assume that the dendrite grows into an infinite volume of liquid, and hence $u(\vec{x}, t) \rightarrow-\Delta$ as $|\vec{x}| \rightarrow \infty \forall t$. Typical experimental values for $\Delta$ range from 0.001 to 0.1 . The length scales involved in the problem are (i) the capillary length $d_{0}$, (ii) a typical scale of the pattern such as the radius of curvature at a tip $\rho$, and (iii) the length scale of the diffusion field $l_{D}$. To fix the ideas, let us consider the measurements of Rubinstein and Glicksman on pivalic acid (PVA) [23]. For a dimensionless undercooling of $\Delta=0.075, \rho=8.5 \mu \mathrm{m}$, and the speed of the tips is $v=390 \mu \mathrm{m} / \mathrm{s}$, which gives a diffusion length $l_{D}=2 D / v=0.38 \mathrm{~mm}$, whereas $d_{0}=3.8 \mathrm{~nm}$. The multiscale character of this situation is obvious: $l_{D}$ and $d_{0}$ differ by five orders of magnitude, and $l_{D}$ is forty times larger than $\rho$. These ratios become even larger for lower undercoolings.

The above equations constitute a notoriously difficult free boundary problem. To simplify the task, theoretical and numerical efforts first concentrated on the treatment of a single needle crystal growing at constant velocity. This situation can be treated by boundary integral methods [20], which are exact in two dimensions (2-d) but have remained approximate in three dimensions (3d). More recently, time-dependent methods have been developed to describe the full growth dynamics [27 29. Of those, the phase-field method [30] seems presently the most compact and precise approach. We use a recent efficient formulation of this method, which has been benchmarked against boundary integral calculations [29]. An "order parameter", or phase-field $\psi(\vec{x}, t)$ is introduced, which is an indicator field distinguishing the solid $(\psi=1)$ and the liquid $(\psi=-1)$ phase. The two-phase system is described by a free energy functional of Ginzburg-Landau type,

$$
\mathcal{F}=\int d V\left[W^{2}(\hat{n})|\vec{\nabla} \psi|^{2}+f(\psi, u)\right],
$$

where $W(\hat{n})$ is the orientation-dependent interface thickness, i.e. the spatial scale on which the phase-field varies smoothly between its equilibrium values $\psi= \pm 1$, and $f(\psi, u)$ is the free energy density. The equations of motion are 


$$
\tau(\hat{n}) \partial_{t} \psi=-\frac{\delta \mathcal{F}}{\delta \psi(\vec{x}, t)},
$$

where $\delta \mathcal{F} / \delta \psi$ denotes the functional derivative, and

$$
\partial_{t} u=D \vec{\nabla}^{2} u+\frac{1}{2} \partial_{t} \psi
$$

The phase-field relaxes to its local minimum free energy configuration, which depends on the local temperature field, with an orientation-dependent relaxation time $\tau(\hat{n})$. The diffusion equation contains a source term to account for the latent heat released or consumed during the phase transformation. For a suitable choice of the functions $f(\psi, u), W(\hat{n})$ and $\tau(\hat{n})$, these equations reduce precisely to the free boundary problem given by Eqs. (2) to (4) in the limit where the interface thickness is small compared to the radii of curvature 29]. A brief description of the model used for our simulations and its relation to the macroscopic free boundary problem is given in the appendix. The key point is that the phase-field equations of motion are partial differential equations which can be integrated on a regular grid on the scale of the dendrite, without knowing explicitly where the solid-liquid interface is located. The phase field rapidly decays to its equilibrium values $\psi= \pm 1$ away from the interface. Therefore, well within the bulk phases, Eq. (10) becomes trivial and Eq. (11) reduces to the ordinary diffusion equation.

\section{DIFFUSION MONTE CARLO ALGORITHM}

\section{A. Outline}

Our goal is to combine the precision of the phase-field method and the efficiency of a DMC treatment for the diffusion field. This is achieved by dividing the simulation domain into an "inner" and an "outer" region as shown in Fig. 11. In the inner region, consisting of the growing structure and a thin "buffer layer" of liquid, we integrate the phase-field equations described above. In the outer region, the diffusion field is represented by an ensemble of random walkers. Walkers are created and absorbed at the boundary between inner and outer domains at a rate which is proportional to the local diffusion flux. The value of the diffusion field in the outer domain is related to the local density of walkers, and the boundary conditions for the integration in the inner region are obtained by averaging this density over coarse-grained boxes close to the boundary. We will now describe in detail the DMC algorithm for the evolution of the random walkers and the connection of the two solutions.

Let us start by recalling some well-known facts about random walkers. Consider first a single point particle performing a Brownian motion in continuous space and time. The conditional probability $P\left(\vec{x}^{\prime}, t^{\prime} \mid \vec{x}, t\right)$ of finding the particle at position $\vec{x}^{\prime}$ at time $t^{\prime}$, given that it started from position $\vec{x}$ at time $t$, is identical to the diffusion kernel,

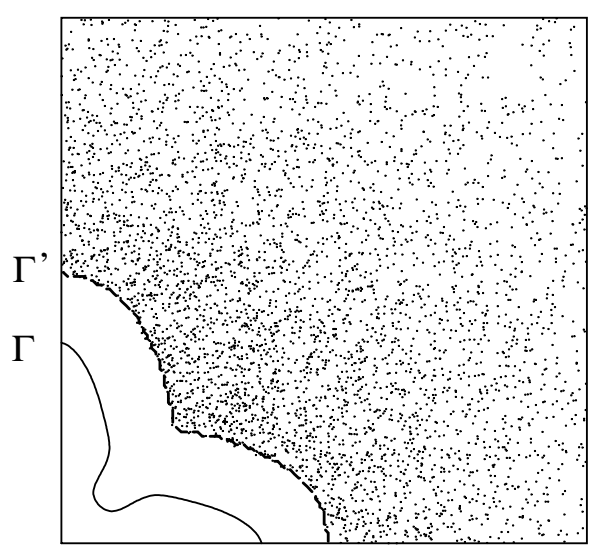

FIG. 1. Simulation of two-dimensional dendritic growth for a dimensionless undercooling $\Delta=0.1$ and a surface tension anisotropy $\epsilon_{4}=0.025$. The solid line is the solid-liquid interface $\Gamma$, the dashed line is the conversion boundary $\Gamma^{\prime}$ between the inner (deterministic) and outer (stochastic) domains, and the dots show the positions of random walkers (only one walker out of 50 is shown for clarity).

$$
P\left(\vec{x}^{\prime}, t^{\prime} \mid \vec{x}, t\right)=\frac{1}{\left[4 \pi D\left(t^{\prime}-t\right)\right]^{d / 2}} \exp \left[\frac{-\left|\vec{x}^{\prime}-\vec{x}\right|^{2}}{4 D\left(t^{\prime}-t\right)}\right],
$$

where $D$ is the diffusion coefficient and $d$ is the spatial dimension. This kernel satisfies the well-known convolution relation

$$
\begin{aligned}
& P\left(\vec{x}^{\prime \prime}, t^{\prime \prime} \mid \vec{x}, t\right)=\int P\left(\vec{x}^{\prime \prime}, t^{\prime \prime} \mid \vec{x}^{\prime}, t^{\prime}\right) P\left(\vec{x}^{\prime}, t^{\prime} \mid \vec{x}, t\right) d \vec{x}^{\prime} \\
& \forall t<t^{\prime}<t^{\prime \prime}
\end{aligned}
$$

Therefore, a realization of a random walk, i.e. the position of a walker as a function of time, represented by a time-dependent vector of real numbers $\vec{x}(t)$, can be obtained on a computer by successive steps. The position of the walker is updated following the scheme

$$
\vec{x}(t+\tau)=\vec{x}(t)+\ell \vec{\xi}
$$

where the components of the random vector $\vec{\xi}$ are independent Gaussian random variables of unit variance. The time increment $\tau$ (not to be confused with the phase-field relaxation time $\tau(\hat{n})$ defined in the preceding section) and the step size $\ell$ must satisfy the relation

$$
\frac{\ell^{2}}{\tau}=2 D
$$

Since time is continuous and Eq. (13) is not restricted to $t^{\prime \prime}-t^{\prime}=t^{\prime}-t$, successive steps may have different time increments (and concomitantly use different step lengths) if Eq. (15) is satisfied for each update. 
The basic idea of Diffusion Monte Carlo simulations is to sample many realizations of diffusion paths. The density of random walkers then satisfies a stochastic differential equation which converges to the deterministic diffusion equation in the limit of an infinite number of walkers. A density of walkers can be defined by a suitable coarse-graining procedure on a scale $L_{c g}$, i.e. by dividing space into cells of volume $L_{c g}^{d}$ and counting the number of walkers within each cell. If the coarse-graining length is chosen larger than the average step length $\ell$, this density evolves smoothly on the scale of $L_{c g}$ over times of order $L_{c g}^{2} / D$, the time for one walker to diffuse through a coarse cell.

From the above considerations, it is clear that the characteristic length and time scales that can be resolved by a stochastic DMC algorithm are set by the step size $\ell$ and the time increment $\tau$, respectively. The key point is that for the present application a high spatial and temporal resolution is needed only close to the interface, whereas far from the dendrite, the coarse-graining length and hence the step size can become much larger than the fine features of the growing crystal. In practice, we choose the step size to be approximately proportional to the distance $d_{c b}$ of the walker from the conversion boundary between the inner (deterministic) and outer (stochastic) regions, i.e.

$$
\ell \approx c d_{c b}
$$

with a constant $c \ll 1$. According to Eq. (15), the time increment between updates grows as the square of the step size, and hence the walkers far from the dendrite have to be updated only rarely. We use dynamical lists to efficiently handle the updating process, as will be described in more detail in Sec. IIIB. For low undercoolings, where the scale of the diffusion field is much larger than the dendrite itself and most of the walkers need only be updated sporadically, we obtain enormous savings of computational time over a straightforward integration of the diffusion equation.

Let us now discuss how the inner and outer regions are interfaced. Two essential goals have to be accomplished. Firstly, we have to supply a boundary condition at the conversion boundary for the integration of the deterministic equations in the inner region, and secondly we need to create and absorb walkers at a rate which is proportional to the local heat flux across this boundary.

The phase-field equations are integrated in the inner region on a regular cubic grid, henceforth called "fine grid", with spacing $\Delta x$. Each node on this grid contains the local values of the phase field $\psi$ and the temperature field $u$. We superimpose on this grid another, coarser grid, of mesh size $L_{c g}=n \Delta x$, such that the links of the coarse grid intersect the links of the fine grid as shown in Fig. 2. The first purpose of this grid is to define the geometries of the two simulation regions and of the conversion boundary. We describe the "state" of each coarse cell by an integer status variable $S_{\alpha \beta \gamma}^{t}$. Here and in the

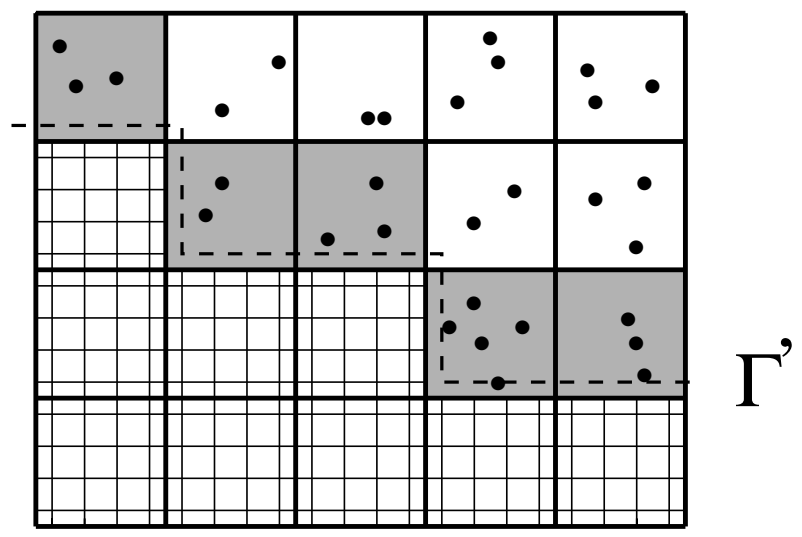

FIG. 2. Sketch of a small part of the conversion boundary in two dimensions for $n=4$. Each cell of the coarse grid (thick lines) contains 16 points of the fine grid (thin lines). The fine grid is shown only in the inner region for clarity. The shaded cells are conversion cells, and walkers are represented by black dots. The boundary $\Gamma^{\prime}$ between inner and outer regions is indicated by a dashed line.

following, greek indices $(\alpha, \beta, \gamma)$ label the cells of the coarse grid along the $x-, y-$, and $z$-directions, whereas latin indices $(i, j, k)$ label the nodes of the fine grid. All cells which contain at least one node of the fine grid where $\psi>0$ are assigned the status "solid" $(S=-2)$. All cells with a center-to-center distance to the nearest solid cell smaller than a prescribed length $L_{b}$ are "buffer cells" $(S=-1)$, whereas all other cells belong to the outer region. Cells of the outer region which have at least one nearest neighbor with buffer status are called conversion cells $(S=0)$ and play the central role in interfacing the two solutions. The dividing surface $\Gamma^{\prime}$ between inner and outer regions is the union of all the links (or plaquettes in three dimensions) of the coarse grid which separate conversion from buffer cells (see Fig. 2). Evidently, as the crystal grows, the geometry of the two regions changes, which means that the status variables must be periodically updated. Details on this procedure are given in Sec. III Q.

We always choose $L_{b}$ sufficiently large to ensure that the phase field is already close to its liquid equilibrium value, $\psi \approx-1$, at the conversion boundary. Hence we can set $\psi=-1$ in the entire outer region and treat only the standard diffusion equation there. In the initial state, the entire system is undercooled to $u=-\Delta$, and no walkers are present. When the crystal grows, it releases latent heat which diffuses away from the interface, and hence the inner region becomes a heat source for the outer region. This heat flux is converted into walkers, each walker representing a certain discrete amount of heat. We define in each coarse cell an integer variable $m_{\alpha \beta \gamma}^{t}$ which contains the number of walkers being within this cell at time $t$. For a specific heat which is independent of temperature, the density of walkers is proportional to the difference between the actual and the initial temperatures, i.e. the temperature in the outer region is related 
to the number of walkers by

$$
u_{\alpha \beta \gamma}^{t}=-\Delta\left(1-\frac{m_{\alpha \beta \gamma}^{t}}{M}\right),
$$

where the constant $M$ fixes the number of walkers in a cell that corresponds to the melting temperature $u=0$.

The inner region is completely delimited by conversion cells. To fix the boundary condition for the integration on the fine grid, it is therefore sufficient to set the field $u$ on all nodes of the fine grid in each conversion cell to the value specified by Eq. (17). The diffusion equation is then timestepped in the inner region using the standard explicit scheme

$$
\begin{aligned}
u_{i j k}^{t+\Delta t}= & u_{i j k}^{t}+\frac{D \Delta t}{(\Delta x)^{2}} \\
& \times\left(u_{i+1 j k}^{t}+u_{i-1 j k}^{t}+u_{i j+1 k}^{t}+u_{i j-1 k}^{t}\right. \\
& \left.\quad+u_{i j k+1}^{t}+u_{i j k-1}^{t}-6 u_{i j k}^{t}\right) .
\end{aligned}
$$

Note that we have omitted for simplicity the source terms due to the phase field, which are zero at the conversion boundary. Seen on a discrete level, this equation can be interpreted as a "pipe flow" equation: the local change of $u$ is given by the sum of the "flow" through all the discrete links ("pipes"), where, for example, the "flow" through a link along $x$ during a timestep is given by $D \Delta t\left(u_{i+1 j k}-u_{i j k}\right) /(\Delta x)^{2}$. For nodes at the boundary of the inner region, some links cross the conversion boundary $\Gamma^{\prime}$, which means that there is exchange of heat with the neighboring conversion cell. This heat flux is collected by the conversion cell and stored in a heat reservoir variable $H_{\alpha \beta \gamma}^{t}$. A symbolic manner to describe the updating of $H_{\alpha \beta \gamma}^{t}$ is

$$
H_{\alpha \beta \gamma}^{t+\Delta t}=H_{\alpha \beta \gamma}^{t}+\frac{D \Delta t}{(\Delta x)^{2}}\left(\sum_{\text {bonds }} u_{\text {grid }}^{t}-u_{c c}^{t}\right),
$$

where the sum runs over all the bonds of the fine grid that cross $\Gamma^{\prime}, u_{\text {grid }}$ is the temperature on a node of the fine grid and $u_{c c}$ is the temperature in the conversion cell given by Eq. (refcoarsetemp). For example, for a conversion cell $(\alpha, \beta, \gamma)$ in contact with a buffer cell $(\alpha-1, \beta, \gamma)$, we have (we recall that the linear dimension of a coarse cell is $\left.L_{c g}=n \Delta x\right)$ :

$$
\begin{aligned}
& H_{\alpha \beta \gamma}^{t+\Delta t}=H_{\alpha \beta \gamma}^{t}+ \\
& \sum_{\substack{\beta=(\beta-1) n+1 \\
\text { with } \quad i=(\alpha-1) n+1}}^{\beta n} \sum_{k=(\alpha-1) n+1}^{\gamma n} \frac{D \Delta t}{(\Delta x)^{2}}\left(u_{i-1 j k}^{t}-u_{i j k}^{t}\right)
\end{aligned}
$$

If the stored quantity of heat exceeds a critical value $H_{c}$ given by

$$
H_{c}=\frac{n^{d} \Delta}{M}
$$

a walker is created at the center of the conversion cell and $H_{c}$ is subtracted from $H_{\alpha \beta \gamma}$. Conversely, if the local heat flux is negative (heat is locally flowing towards the dendrite) and $H_{\alpha \beta \gamma}$ falls below $-H_{c}$, a walker is removed from the cell and $H_{c}$ is added to the reservoir. This algorithm exactly conserves the total heat if the contributions of the fine grid, the reservoir variables and the walkers are added. In dimensional quantities, each walker is equivalent to an amount of heat $\Delta Q$ equal to

$$
\Delta Q=\frac{L(n \Delta x)^{d} \Delta}{M} .
$$

The walkers are restricted to the outer region. If a walker attempts to jump across the conversion boundary, the move is discarded and the walker stays at its old position until the next update. If $c$ in Eq. (16) is small enough, such jumps are attempted almost only by walkers close to the conversion boundary. Accordingly, this procedure is a convenient way of implementing the re-absorption of walkers: if a walker stays in a conversion cell, the heat flux is more likely to be directed towards the inner region, which increases the chances for the walker to be absorbed. An alternative method, namely to deposit all the heat contained in a walker in the fine grid and remove the walker upon its crossing of the boundary, would create stronger temperature fluctuations on the fine grid close to the conversion boundary.

In summary, the conversion process is handled using three auxiliary fields on the coarse grid: the status field $S_{\alpha \beta \gamma}$ which encodes the geometry of the buffer layer and the conversion boundary, the field $m_{\alpha \beta \gamma}$ that contains the number of walkers in each cell and is zero in the inner region, and the heat reservoir field $H_{\alpha \beta \gamma}$, which is different from zero only in conversion cells. Let us comment on the size of the grids and the resulting memory usage. The fine grid needs to be large enough to accommodate the dendrite and the liquid buffer layer during the whole time of the simulation. Especially in three dimensions, the restrictions on storage space make it necessary to fully use the fine grid. The coarse grid needs to cover at least the same space region as the fine grid. As will be detailed below, for an efficient handling of the walkers close to the conversion boundary, it is desirable to always have some portion of coarse grid in front of the conversion boundary, and hence the coarse grid should actually cover a slightly larger region of space than the fine grid. Since the coarse grid has far less nodes than the fine grid (1 node of coarse grid for $n^{d}$ nodes of fine grid), this does not significantly increase the storage requirement. In addition, we need an array to store the positions of the walkers. The latter are represented by "continuous" positions and need no grid for their evolution. The walkers can therefore leave the region of space where the grids are defined and diffuse arbitrarily far away from the dendrite, allowing us to simulate growth into an infinite medium. The most storage-intensive part is the fine grid. In fact, the limiting factor for most of our three-dimensional simulations 
is not so much computation time, but rather the storage space needed to accommodate large dendrites.

Finally, let us describe how the different parts of the algorithm are connected. The program runs through the following steps:

1. Setup (or update) the status field $S_{\alpha \beta \gamma}$ on the coarse grid to fix the geometry of the conversion boundary

2. Calculate the temperature in each conversion cell and set the boundary condition for the inner region on the fine grid

3. Timestep the phase-field equations on the fine grid and calculate the heat flux between the inner region and the conversion cells

4. Update the heat reservoir variables $H_{\alpha \beta \gamma}$ and create or absorb walkers in the conversion cells

5. Advance the walkers

6. Repeat steps 2 through 5. From time to time, extract the shape of the dendrite and store it for future processing. If the phase boundary has moved by more than a coarse cell size, go back to step 1 .

In the following subsections, we will give more details on some features of our implementation, such as the updating of the walkers, the updating of the geometry, the choice of parameters, and parallelization.

\section{B. Updating random walkers}

Before going into details, let us briefly point out similarities and differences between our method and other DMC algorithms. Such methods are widespread in Quantum Monte Carlo (QMC) calculations where they are used to solve the Schroedinger equation in imaginary time [7. Each walker represents a configuration in a usually highdimensional Hilbert space, and the density of walkers is proportional to the square amplitude of the wave function. In contrast, in our method the walkers evolve in real space, and their density represents the temperature field. The most important difference, however, is that in QMC all walkers are usually updated at the same time, whereas in our method some walkers are updated much more rarely than others. Therefore, it would be very inefficient to visit every walker in each timestep. Instead, we work with dynamical lists.

To simplify the bookkeeping of the different update times, we enforce that updating takes place only at the discrete times when the fine grid is updated, i.e. for $t=i \Delta t, i=1,2, \ldots$. Then, we can make a list for every timestep containing all the walkers that have to be updated at that moment. However, these lists greatly vary in length and can therefore not easily be accommodated in standard arrays of variables. Therefore, we

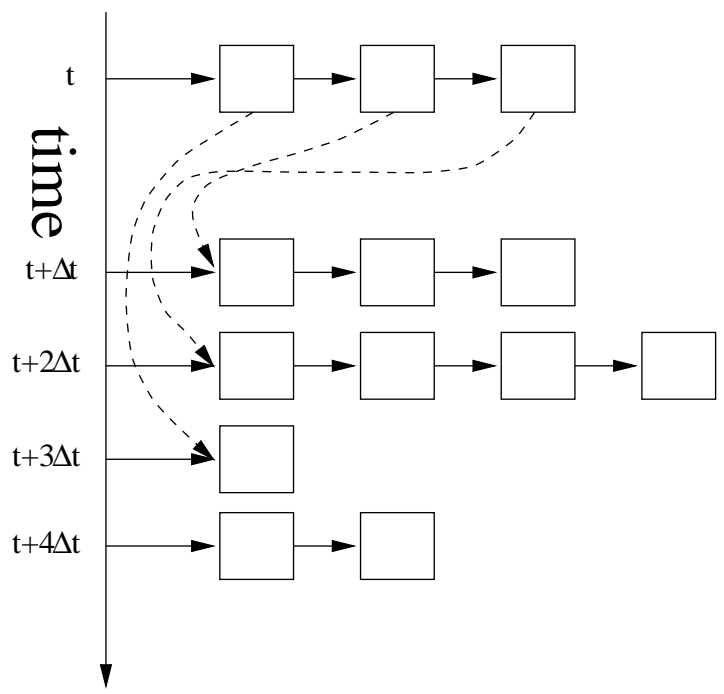

FIG. 3. Sketch illustrating the configuration of the dynamical walker lists. Each box stands for a walker, and the full arrows indicate pointer variables; the "backbone" array of pointers is represented by the downward arrow on the left. At time $t$, walkers are updated and prepended to the lists corresponding to their next update time, as indicated by broken arrows.

define a data structure that contains the coordinates of one walker plus a pointer variable. Within a given list, the pointer associated with one walker indicates the next element of the list, or contains an end of list tag if the corresponding walker is the last one of the list. An array of pointer variables indicates for each timestep the first element of the corresponding list. This array is the "backbone" of the list structure. It is easy to add new walkers to a list: the pointer of the new walker is set to the former first element of the list, and the pointer of the backbone is set to the new walker (see Fig. 3). Lists of arbitrary length can be constructed, and every walker is visited only when it actually has to be updated.

At a given time $t$, the program works through the corresponding list of walkers. The treatment of each walker starts by looking up the status of the coarse grid cell corresponding to its position. If the walker is inside a buffer cell because the conversion boundary has moved since its last update, it is removed. This removal does not violate heat conservation because the heat associated with the walker is accounted for in the initialization of the temperature field inside newly created buffer cells (see Sec. III Q, Eq. (24) below). If it is inside a conversion cell, and the corresponding reservoir variable $H_{\alpha \beta \gamma}^{t}<-H_{c}$, the walker is removed and $H_{c}$ is added to the reservoir. In all other cases, the jump distance $\ell$ and corresponding time increment are determined and a new position is selected according to Eq. (14). To apply Eq. (16) for the jump distance $\ell$, we need to determine the distance of a walker to the conversion boundary. It would be very inefficient to calculate this distance for each walker separately, especially when the shape of the boundary be- 
comes complex. Therefore, we use the status field $S_{\alpha \beta \gamma}^{t}$ on the coarse grid in the outer region to store an approximate value for this distance, which can then be easily looked up by each walker before a jump. Some more details are given in Sec. IIIC.

As mentioned above, we restrict the walker updating to a discrete set of times. Therefore, the time increment $\tau$ in Eq. (14) has to be an integer multiple of the time step $\Delta t$, which would not be the case if we directly applied Eqs. (16) and (15). We solve this problem by defining a lower cutoff for the jump distances,

$$
\ell_{\min }=\sqrt{2 D n_{t} \Delta t}
$$

where $n_{t}$ is a fixed integer, and replace the jump distances $\ell$ found from Eq. (16) by the closest integer multiple of $\ell_{\text {min }}$. We also define a maximum jump length $\ell_{\max }$, mainly to limit the size of the backbone pointer array: with a maximum jump distance $\ell_{\max }$, each walker is at least updated every $\ell_{\max }^{2} /(2 D \Delta t)$ timesteps. Consequently, the discrete time modulo this number can be used to index the pointer variables in the backbone array.

It should be mentioned that in our list structure, it is difficult to find a walker which is close to a given position, because all sublists must be searched. This is important because the number of walkers in the conversion cells has to be known for the interfacing with the inner solution. To avoid time-consuming sweeps through the walker lists, we update the walker number field $m_{\alpha \beta \gamma}^{t}$ on the coarse grid whenever a walker jumps.

\section{Updating the geometry}

We now describe more in detail how the status field on the coarse grid is setup and adapted to the changing geometry. When the dendrite grows, the configuration of the buffer layer and the conversion boundary has to change in order to maintain a constant thickness $L_{b}$ of the buffer layer. Cells which are part of the outer region at the beginning of the simulation may become conversion cells, then part of the buffer layer, and finally part of the dendrite. Under the conditions we want to simulate, the crystal may locally melt back, but no large regions of space will undergo the transition from solid to liquid, and hence we do not consider the inverse status change (from buffer to conversion cell, for example). Typically, at low undercoolings a readjustment of the geometry becomes necessary only after 1000 to 10000 timesteps. Therefore, the efficiency requirements are not as stringent as in the other parts of the program.

The procedure starts with a sweep through the fine grid. Every cell of the coarse grid which contains at least one node of the fine grid where $\psi>0$ is assigned the status "solid" $\left(S_{\alpha \beta \gamma}^{t}=-2\right)$. Next, the solid cells at the boundary of the dendrite (i.e. each solid cell which has at least one neighboring cell which is not solid) are used to define the buffer region: all cells with a center-tocenter distance less than $L_{b}$ of a boundary cell which are not solid are assigned the status "buffer" $\left(S_{\alpha \beta \gamma}^{t}=-1\right)$. When a conversion cell or a cell of the outer region becomes a buffer cell, we need to define the initial values of the two fields on the fine grid. The phase field is set to its liquid value, $\psi=-1$. The temperature is calculated from the total heat contained in the cell, taking into account both the walkers and the heat reservoir variables in the conversion cells in order to ensure that the total amount of heat remains conserved, i.e.

$$
u_{\text {init }}=\frac{\Delta}{M}\left(m_{\alpha \beta \gamma}^{t}+H_{\alpha \beta \gamma}^{t} / H_{c}\right) .
$$

All nodes of the fine grid within the new buffer cell are initially assigned this value. The walkers contained in the cell are removed.

All cells of the outer region which are adjacent to the buffer, i.e. which have at least one neighbor with buffer status, are conversion cells $\left(S_{\alpha \beta \gamma}^{t}=0\right)$. When a cell of the outer region becomes a conversion cell, its heat reservoir variable is initialized at zero.

Finally, in the outer region, which is comprised of all the other cells, the status field is used to store an approximate value for the distance from the conversion boundary. A precise determination of this distance is rather costly in computation time, because for each cell in the outer region, we must calculate the distance to all conversion cells and retain the minimum value. A much cheaper, albeit approximate method is the following. As mentioned, in a conversion cell we have $S_{\alpha \beta \gamma}^{t}=0$. We assign to all cells adjacent to a conversion cell the value $S_{\alpha \beta \gamma}^{t}=1$. Neighbors of the latter receive the value $S_{\alpha \beta \gamma}^{t}=2$, and we continue this process outward by assigning the value $S_{\alpha \beta \gamma}^{t}=i+1$ to all cells adjacent to a cell with $S_{\alpha \beta \gamma}^{t}=i$. For a relatively simple geometry such as a single growing dendrite, the status field can be correctly set up on the whole lattice during a single outward sweep, starting from the center of the dendrite. The number assigned to a given cell can be used as a measure for the distance. Note that the exact relationship of the number to the distance depends on the direction with respect to the axes of the coarse grid; our numerical tests below show, however, that this anisotropy in the distance function does not significantly influence the dendrite shapes.

If we follow this procedure, the coarse grid needs to cover the entire region of space where the jump distance varies. Even though we introduce a large-scale cutoff $\ell_{\max }$, this would become prohibitive in terms of memory usage for truly multi-scale problems. Fortunately, such a sophisticated scheme for the determination of the distance is mainly needed close to the dendrite (for example, a walker that enters in the space between two dendrite arms needs to make small steps). Once a walker has left the vicinity of the dendrite, this rather complicated estimate for the distance to the conversion boundary can 
be replaced by a simpler one, for example the distance to the closest dendrite tip. In consequence, the coarse grid needs to cover only a slightly larger region of space than the fine grid.

Finally, let us comment on the integration of the phasefield equations in the inner region. We need to know which part of the fine grid must be timestepped. This information is encoded in the status field $S_{\alpha \beta \gamma}^{t}$ on the coarse grid. It would, however, be rather inefficient in terms of memory access time to integrate the inner region "coarse cell by coarse cell". Instead, integration proceeds along the spatial direction corresponding to successive memory locations, which is the $\mathrm{x}$-direction in our implementation. During the updating of the status field, the program determines for each $y$ and $z$ coordinate the range(s) to be integrated along $x$ and keeps this information in a lookup table. This table is updated every time the status field changes.

\section{Choice of computational parameters}

There are number of parameters in our algorithm which can be adjusted to maximize the computational efficiency. However, certain restrictions apply. Firstly, there are various length scales. In order of increasing magnitude, those are:

1. the lattice spacing of the fine grid, $\Delta x$,

2 . the minimum jump length of the walkers, $\ell_{\min }$,

3. the size of a coarse-grained cell, $L_{c g}=n \Delta x$, and

4. the buffer thickness $L_{b}$.

The minimum jump length should be of the order of the inner grid spacing to assure a precise interfacing between inner and outer solutions. On the other hand, a larger $\ell_{\text {min }}$ means less frequent walker updating. We usually worked with $\ell_{\min } \approx 2 \Delta x$, or $n_{t} \approx 10$ in Eq. 23. On the other hand, $\ell_{\min }$ has to be smaller than $L_{c g}$ in order to achieve a well-defined coarse-graining. The coarsegraining length, in turn, is limited by geometrical constraints. The conversion boundary appears "jagged" on the scale of $L_{c g}$ (see Fig. 2). In order to render the effects of this coarse geometry irrelevant for the interface evolution, the buffer thickness must be much larger than this scale, $L_{b} \gg L_{c g}$. We found that $L_{c g} \approx 0.1 L_{b}$ is sufficient to achieve this goal. In our simulations, we mostly worked with $n=4\left(L_{c g} \approx 2 \ell_{\min }\right)$ and $n=8$ for larger buffer sizes.

Next, consider the constant of proportionality $c$ between the walker jump length and the distance to the conversion boundary, $d_{c b}$. Since the Gaussian random vector $\vec{\xi}$ in Eq. (14) has no cutoff, steps of arbitrary length are possible, and hence even a walker which is far away can jump directly to the conversion boundary. The number of such events has to be kept small, because otherwise the conversion process is influenced by the far field with its coarse length and time scales. This goal can be naturally achieved by choosing $c$ small enough. For example, for $c=0.1$, only jumps with a length of more than 10 standard deviations can reach the conversion boundary, which represents a negligible fraction. On the other hand, the increase of $\ell$ with distance determines the efficiency of the algorithm, and hence $c$ should be chosen as large as possible. We usually worked with $c=0.1$, which seems to provide a good compromise.

Finally, the parameter $M$ determines the number of walkers per coarse cell and hence the precision of the stochastic representation for the temperature field and the diffusion equation. Considering Eq. (17), we see that the temperature at the boundary of the inner region takes discrete values spaced by $\Delta / M$. In addition, for a homogeneous distribution of walkers in a system at $u=0$, the temperature fluctuations are of order $\Delta / \sqrt{M}$. On the other hand, increasing $M$ means longer computation time because more random walks have to be performed. In addition, the total number of walkers $N$ necessary to simulate a dendrite of final volume $V$ is

$$
N=\frac{M V}{(n \Delta x)^{d} \Delta},
$$

which means that high values of $M$ become prohibitive, especially at low undercooling. Fortunately, a good precision of the solution can be obtained also by increasing $L_{b}$, as will be described in Sec. IV. In practice, we worked with values of $M$ ranging between 25 and 100 .

\section{E. Boundary conditions and symmetries}

For a two-dimensional dendrite seeded at the origin and with arms growing along the $x$ - and $y$-directions, the simulations can be accelerated by taking advantage of the cubic symmetry. There are several symmetry axes, and consequently it is sufficient to integrate the equations in a part of the plane while imposing reflective boundary conditions at the proper axes to enforce the symmetry. These boundary conditions have to be imposed both on the fine grid and for the walkers. For the symmetry axes at $x=0$ and $y=0$, this can be easily achieved by choosing one of the nodes of the coarse grid to coincide with the origin. Then, the two symmetry axes coincide with bonds in the coarse grid. On the fine grid, the nodes outside the simulation domain but adjacent to the boundary are set to the values of their mirror images inside the simulation domain after each timestep. Walkers that attempt to cross the boundaries are reflected, i.e. instead of their "true" final position outside the simulation domain, its mirror image with respect to the symmetry axis is chosen. Another interpretation of this boundary condition for the walkers is to imagine that there exists an ensemble of "mirror walkers" which are the images of 


\section{"mirror walker"}

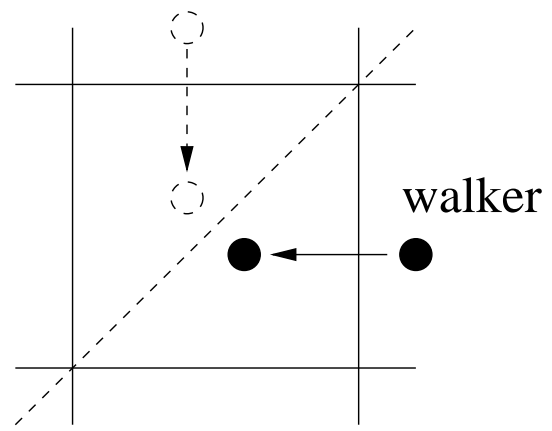

FIG. 4. Sketch illustrating the implementation of reflecting boundary conditions at the symmetry axis $x=y$. Shown is a cell of the coarse grid (solid lines) on the diagonal $x=y$ (dashed line). A walker inside the simulation domain $(x>0$, $0<y<x)$ enters the cell. An accompanying "mirror walker" (open circles), the image of the walker with respect to the symmetry axis, enters the same cell.

the walkers inside the simulation domain. When a walker jumps outside of the simulation domain, its mirror image jumps inside, and interchanging the walker and its mirror, we just obtain a reflection of the walker at the boundary as above.

The latter view is useful when considering the last symmetry axis, the diagonal $x=y$. While the boundary conditions on the fine grid and for the walkers can be implemented as before, the conversion process requires special attention, because the symmetry axis does not coincide with the boundaries of a coarse cell. When a walker enters a coarse cell situated on the diagonal, there is an additional "mirror walker" entering the same coarse cell (see Fig. 4), and hence the number of walkers $m_{\alpha \beta \gamma}^{t}$ has to be increased by two (or, equivalently, decreased by two if a walker leaves the cell). Similarly, walkers are created and absorbed in pairs, which means that walker creation in such a cell can occur only when the heat reservoir exceeds twice the equivalent of one walker. In addition, when calculating the heat flux received by conversion cells on the diagonal, both the "real" and the "mirror" flux has to be taken into account. It is clear that this procedure induces an anisotropy in the conversion process; our tests showed, however, that its effect is undetectable for reasonable buffer thickness.

In three dimensions, the reduction in computational resources is even more dramatic. For example, using the symmetry planes $y=0, x=y$, and $x=z$, i.e. integrating only the domain $x>0,0<y<x, z>x$, we need only integrate $1 / 48$ of the full space, i.e. one eighth of one dendrite arm. The planes $x=y$ and $x=z$ can be handled as described above, with the exception of cells on the diagonal $x=y=z$. Such cells actually have only $1 / 6$ of their volume within the simulation domain, and for each walker entering a cell, there are 5 mirror walkers to be considered.

\section{F. Parallelization}

Even though our algorithm is very efficient as will be shown below, the demands on computation time and RAM storage space rapidly increase when the undercooling is lowered. Therefore, we have developed a parallel version of our code for the Cray T3E at the National Energy Research Scientific Computing Center (NERSC), using the shared memory library SHMEM.

We are mainly interested in the development of a single primary dendrite branch. Hence, an efficient method of parallelization is to divide the simulation domain in "slices" normal to the growth direction, and to distribute the slices among the processors. In the inner region, the integration of the partial differential equations makes it necessary to exchange the boundary values between neighboring processors after each timestep. This is a standard procedure. The more delicate points are the handling of the walkers and the updating of the geometry.

Each processor stores only the parts of the fine grid it has to integrate, along with the values of the status field in the whole simulation domain. The latter is necessary to correctly handle the walkers. For the walkers which are far from the dendrite, the average jump distance may become much larger than the thickness of a computational slice. But if a walker approaches the conversion boundary, the conversion process has to be handled by the "local" processor which contains the appropriate part of the fine grid. Therefore, the walkers need to be redistributed after their jumps. We have found it sufficient to implement "exchange lists" between neighboring processors, i.e. processors which contain adjacent parts of fine grid. If a walker jumps to a position outside of the local slice, it is stored in one of two lists, corresponding to "upward" and "downward" motion. After each timestep, these lists are exchanged between neighboring processors. As most of the walkers make several small steps before reaching the conversion boundary, this procedure assures the correct redistribution of walkers with insignificantly few errors, which arise in the rare case that a walker arrives at the conversion boundary after several large jumps.

The only step of the algorithm which needs massive exchange of data between the processors is the updating of the geometry: each processor has to determine locally the "solid" part of its computation domain, and this information has to be exchanged in order to correctly setup the whole status field on each processor. However, as mentioned earlier, the geometry is updated only rarely, and therefore this part of the algorithm does not represent a significant computational burden. We have found that the parallel version of our code showed satisfactory execution time scaling when the number of processors is increased. 
TABLE I. Computational parameters for the benchmark simulations in two dimensions.

\begin{tabular}{l|c|c}
\hline \hline Quantity & Symbol & Value \\
\hline Interface thickness & $W_{0}$ & 1 \\
Anisotropy & $\epsilon_{4}$ & $0.025666,0.000666$ \\
Effective Anisotropy & $\epsilon_{4}^{e}$ & $0.025,0.0$ \\
Relaxation time & $\tau_{0}$ & 1 \\
Kinetic anisotropy & $\delta_{4}$ & 0 \\
Grid spacing & $\Delta x$ & 0.4 \\
Timestep & $\Delta t$ & 0.003 \\
Diffusion coefficient & $D$ & 10 \\
Coupling constant & $\lambda$ & 15.957 \\
Capillary length & $d_{0}$ & 0.0554 \\
Kinetic coefficient & $\beta_{0}$ & 0 \\
Undercooling & $\Delta$ & 0.3 \\
Coarse cell size & $n$ & 4 \\
Number of walkers per coarse cell & $M$ & 50 \\
\hline \hline
\end{tabular}

\section{NUMERICAL TESTS}

The accuracy of the standard phase-field method has been assessed in detail by comparison to boundary integral results 29. Therefore, to test the stochastic algorithm it is sufficient to check its results against direct simulations of the standard deterministic phase-field equations. The most critical questions are whether the use of the rather coarse lattice for the conversion introduces spurious anisotropy, and what is the magnitude of the temperature fluctuations generated by the stochastic treatment of the far field. The main parameters which control both of these effects are the thickness of the buffer layer and the number $M$ of walkers generated per coarse cell. The boundary condition for the inner region is imposed on a coarse geometry with a cutoff scale of $n \Delta x$, and the temperature at the boundary is a stochastic variable which changes as walkers are created, absorbed, enter, or leave a conversion box, and which assumes discrete values spaced by $\Delta / M$. When the buffer layer is much larger than the size of a coarse cell, $L_{b} \gg n \Delta x$, the field is "smoothed out" in space and time by the diffusive dynamics. We expect high spatial and temporal frequencies to decay rapidly through the buffer layer, and hence the evolution of the interface to become smoother as $L_{b}$ is increased.

We conducted two-dimensional simulations at an intermediate undercooling, $\Delta=0.3$. At this value of $\Delta$, the standard phase-field method can still be used to simulate non-trivial length and time scales of dendritic evolution, but the length scale of the diffusion field is large enough to provide a serious test for the random walker method, i.e. the diffusion length is much larger than the thickness of the buffer layer. Table I shows the computational parameters that were used for these tests. Only the first quadrant was simulated, with reflecting boundary conditions at $x=0$ and $y=0$. Fig. 5(a) shows a comparison
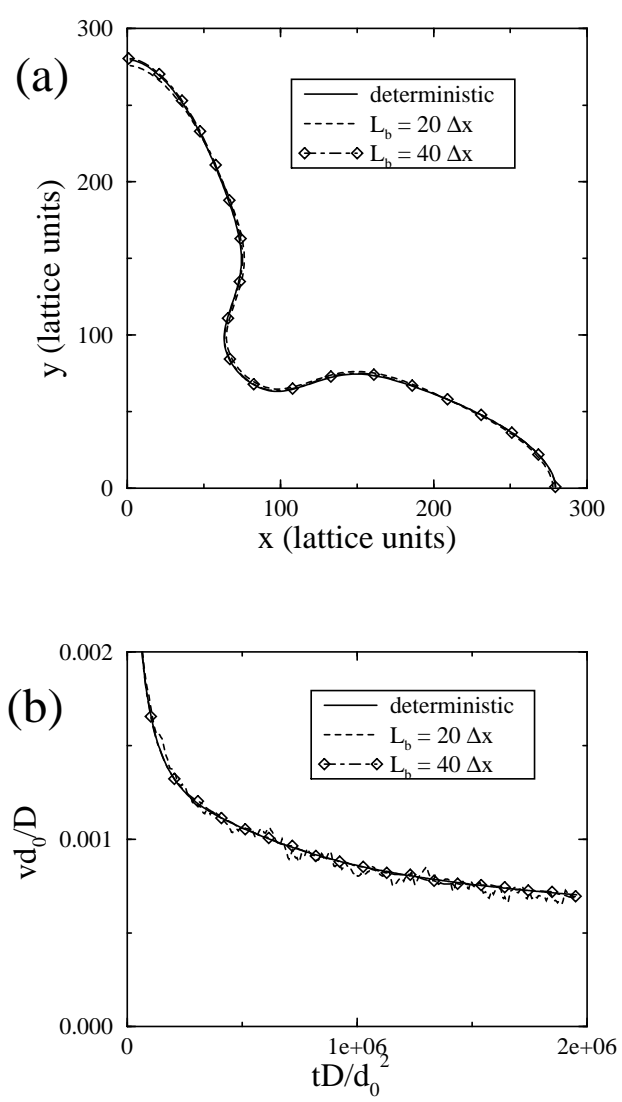

FIG. 5. Comparison of standard (deterministic) phase-field and random walker method in two dimensions for $\Delta=0.3$ and $\epsilon_{4}=0.025$. (a) Dendrite shapes, represented by the contour line $\phi=0$, after 200000 iterations, (b) tip velocity versus time. 


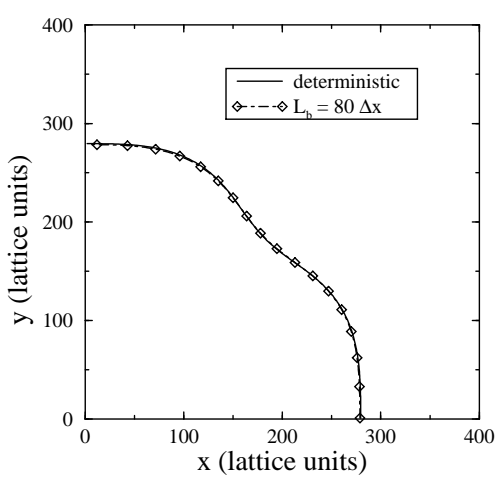

FIG. 6. Comparison of "dendrite" shapes without fourfold anisotropy after 500000 iterations.

of dendrite shapes obtained from the standard phase-field and from our algorithm with different buffer sizes. While the shapes slightly differ for $L_{b} / \Delta x=20$, the curve for $L_{b} / \Delta x=40$ is almost undistinguishable from the deterministic shape. Fig. 5(b) shows the velocity of the dendrite tip along the $x$-direction, measured over periods of 500 iterations, versus time. The fluctuations around the deterministic value are much larger for $L_{b} / \Delta x=20$ than for $L_{b} / \Delta x=40$, and for $L_{b} / \Delta x=80$ (not shown) the curve obtained from the stochastic method is very close to the deterministic data. For comparison, the diffusion length $2 D / v$ at the end of the run is about $400 \Delta x$.

A particularly sensitive test for the anisotropy of the conversion process is the growth of a circular germ without anisotropy, because such a germ is unstable against even smallest perturbations. This can be clearly seen from Fig. 6: even though we completely screen the fourfold anisotropy created by the lattice $\left(\epsilon_{4}^{e}=0\right)$, the weak next harmonic of the lattice anisotropy, proportional to $\cos 8 \theta$, destabilizes the circle and leads to the formation of bulges in the (21)- and (12)-directions. For $L_{b} / \Delta x=80$, the stochastic algorithm perfectly reproduces this trend, and we can hence conclude that the anisotropy created by the coarse structure of the conversion boundary is negligibly small. Note that the diffusion field extends to a distance of more than 1000 lattice units at the end of this run, which means that the larger part of the simulation domain is integrated by the stochastic method.

To quantify the numerical noise, we performed 2-d simulations of the simple diffusion equation in a system of $N \times N$ lattice sites with $N=160$. One half of the system $(x<0)$ was integrated by the stochastic algorithm, whereas in the other half $(x>0)$ we used a standard Euler algorithm. The conversion boundary $\Gamma^{\prime}$ hence coincides with the $y$-axis, and there is a single column of conversion cells along this axis. We used $\Delta x=1, \Delta t=0.02$, $D=1, \Delta=1$, and applied no-flux boundary conditions at $x= \pm N / 2$ and periodic boundary conditions along $y$. The system was initialized at $u=0$ everywhere, i.e. in the walker region we randomly placed $M$ walkers in each coarse cell. When the walkers evolve, fluctuations

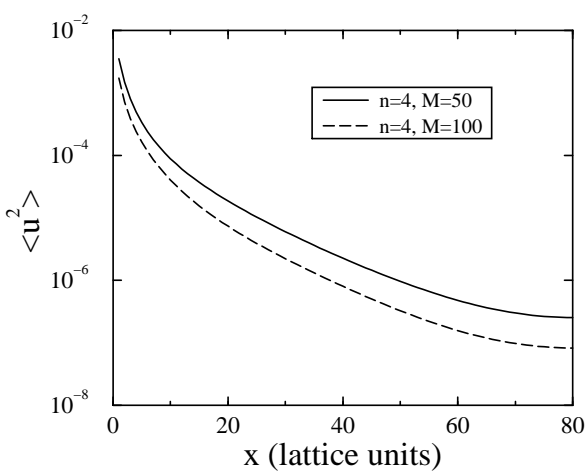

FIG. 7. Variance of temperature fluctuations, $\left\langle u^{2}\right\rangle$, as a function of the distance from the conversion boundary for two values of the walker parameter $M$.

are created in the deterministic region, which plays the role of the buffer layer. We recorded $u^{2}$ as a function of $x$ and averaged over a time which is long compared to the diffusive relaxation time of the system, $N^{2} / D$. The results for two different choices of the walker parameter $M$ are shown in Fig. 7. In an infinite homogeneous system filled with walkers, the distribution of the number of walkers in a given coarse cell is Poissonian, which means that the fluctuations in the walker numbers are of order $\sqrt{M}$. If this scaling remains valid for the conversion cells in our hybrid system, we expect $\left\langle u^{2}\right\rangle \sim 1 / M$ close to the conversion boundary, which is indeed well satisfied. As shown in Fig 7, the variance of the temperature fluctuations rapidly decreases with the distance from the conversion layer - by four orders of magnitude over the distance of 80 lattice sites. No simple functional dependence of $\left\langle u^{2}\right\rangle$ on $x$ is observed. We expect high spatial and temporal frequencies to be rapidly damped. A theoretical calculation of $\left\langle u(x)^{2}\right\rangle$ seems possible but non-trivial because the random variables which are the sources of the fluctuations in the deterministic region are correlated in space and time by the exchange of walkers through the stochastic region and the diffusion of heat through the deterministic region. For our present purpose, we can draw two important conclusions. Firstly, for a reasonable thickness of the buffer layer, fluctuations are damped by several orders of magnitude. The residual fluctuations are much smaller than the thermal fluctuations represented by Langevin forces that have to be introduced in the equations of motion to observe a noticeable sidebranching activity [31]. Indeed, for sufficiently large buffer layers we always observe needle crystals without sidebranches. Secondly, the fluctuations at the solid-liquid interface can be reduced both by increasing the number of walkers and by increasing the thickness of the buffer layer, which allows to accurately simulate dendritic evolution with a reasonable number of walkers.

In Table III, we compare the run times of our code on a DEC Alpha $533 \mathrm{MHz}$ workstation along with the run time of the deterministic phase field reference simulation. 
TABLE II. Execution times of the benchmark simulations for various sets of computational parameters.

\begin{tabular}{c|c|c|c}
\hline \hline & $M$ & $L_{b} / \Delta x$ & CPU time $(\mathrm{min})$ \\
\hline Deterministic & - & - & 1950 \\
& 50 & 20 & 89 \\
& 50 & 40 & 110 \\
& 100 & 40 & 119 \\
\hline \hline
\end{tabular}

The gain in computational efficiency is obvious. Increasing the buffer layer from $L_{b}=20 \Delta x$ to $L_{b}=40 \Delta x$ reduces the amplitude of the temperature fluctuations at the solid-liquid interface by more than an order of magnitude, whereas the computation time increases by only $25 \%$. Comparing the runs with different values of $M$, we see that the walker part of the program accounts only for a small part of the total run time.

From these results, we can conclude that the computational effort that has to be invested to simulate a given time increment scales approximately as the size of the fine grid region, i.e. as the size of the dendrite. This is a major advance with respect to the standard phase-field implementation on a uniform grid, where the computation time scales with the volume enclosing the diffusion field. The spatial and temporal scales of dendritic evolution that can be simulated with our method are hence limited by the integration of the phase-field equations on the scale of the dendrite.

All the data shown so far are for two-dimensional simulations. We repeated similar tests in three dimensions and obtained comparable results for the quality of the solution and the efficiency of the code. We will not display the details of these comparisons here, but rather show an example of a three-dimensional simulation under realistic conditions to demonstrate that our method is capable of yielding quantitative results in a regime of parameters that was inaccessible up to now. In Fig. 8, we show snapshot pictures of a three-dimensional dendrite growing at an undercooling of $\Delta=0.1$ and for a surface tension anisotropy $\epsilon_{4}^{e}=0.025$, which is the value measured for PVA 24]. The other computational parameters are $W_{0}=1, \Delta x=0.8, \epsilon_{4}=0.0284, \tau_{0}=0.965, \delta=0.0364$, $n=4, M=50, L_{b} / \Delta x=48, D=24, \Delta t=0.004$, and $\lambda=39.6$ (giving $d_{0}=0.0223, \beta_{0}=0$ ), and the simulation was started from a homogeneously undercooled melt with an initial solid germ of radius $r=2 \Delta x$ centered at the origin. During the run, we recorded the velocity $v(t)$ and the radius of curvature $\rho(t)$ of the dendrite tip. The latter was calculated using the method described in Ref. [29]. With these two quantities, we can calculate the time-dependent tip selection parameter

$$
\sigma^{*}(t)=\frac{2 D d_{0}}{[\rho(t)]^{2} v(t)}
$$

The results are shown in Fig. 9. In the initial stage

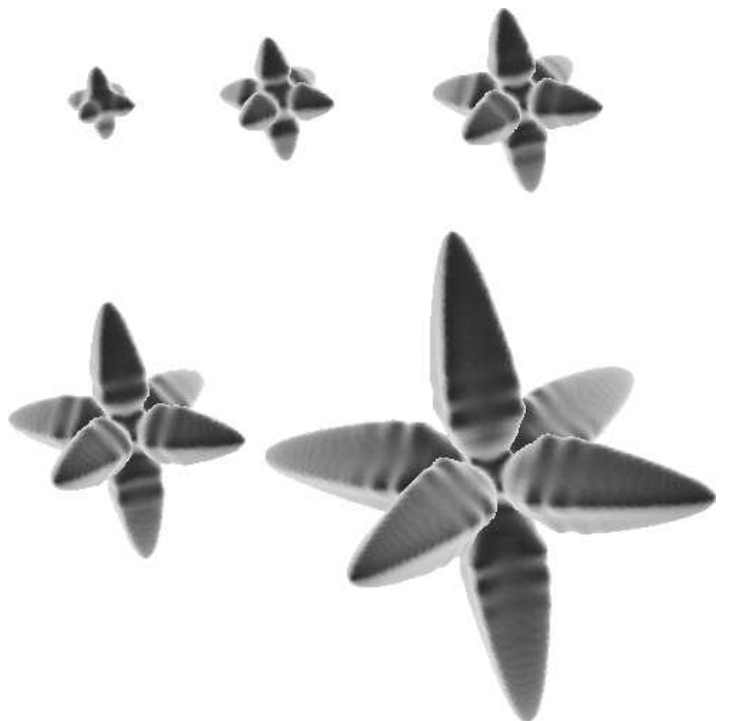

FIG. 8. Snapshots of a three-dimensional dendrite at $\Delta=0.1$ after 60000, 120000, 200000, 300000, and 650000 timesteps (from top left).

during which the arms emerge from the initial sphere, growth is very rapid. Subsequently, the tips slow down while the diffusion field builds up around the crystal. At the end of the run, the velocity has almost converged to a constant value that is in excellent quantitative agreement with the velocity predicted by the boundary integral solution of the sharp-interface steady-state growth equations assuming an axisymmetric surface energy and tip shape (i.e. the most accurate numerical implementation of solvability theory to date [32]). This velocity is also in reasonably good agreement with the velocity predicted by the linearized solvability theory of Barbieri and Langer [33], even though the actual tip radius in both the phase-field simulation and the boundary integral calculation differ from the tip radius of the paraboloidal shape assumed in this theory. A more detailed discussion of this point and the entire steady-state tip morphology can be found in Ref. [18].

Remarkably, the selection constant $\sigma^{*}$ becomes almost constant long before the velocity and the tip radius have reached their steady-state values. This is in good agreement with the concepts of solvability theory, which stipulates that the selection of the tip parameters is governed by the balance between the anisotropic surface tension and the local diffusion field at the tip. To establish the correct local balance, diffusion is necessary only over a distance of a few tip radii, whereas the buildup of the complete diffusion field around an arm requires heat transport over the scale of the diffusion length, $D / v$. Our simulation shows that $\sigma^{*}$ indeed becomes essentially constant soon after the formation of the primary arms. This fact can be used to derive scaling laws for the evolution of the dendrite arms at low undercooling during the transient that leads to steady-state growth [17]. Finally, even at the end of the simulations, where the dendrite arms 


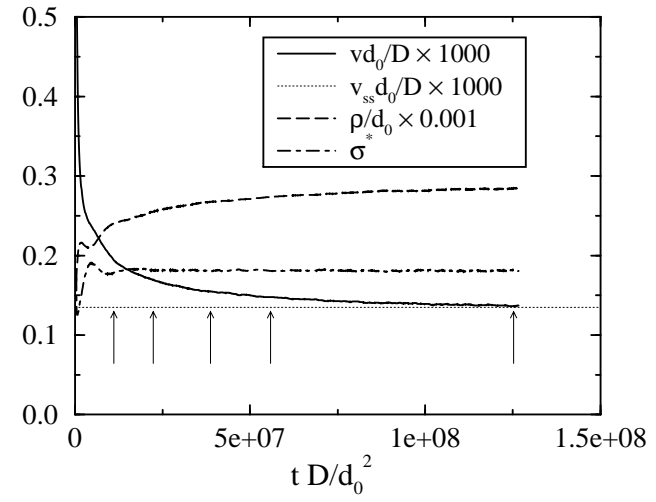

FIG. 9. Tip velocity, tip radius and selection parameter versus time for the run of Fig. 8. Arrows mark the times of the snapshot pictures. Length and time are rescaled by $d_{0}$ and $d_{0}^{2} / D$, respectively. The steady-state velocity $v_{s} s$ was calculated using a boundary integral method [32].

are well developed, no sidebranches are visible. We repeated the same simulation for different thickness of the buffer layer, and observed no changes in the morphology. Tiny ripples can in fact be seen close to the base of the dendrite shaft, but the amplitude of these perturbations does not depend on the noise strength. We therefore believe that this is rather a deterministic instability due to the complicated shape of the dendrite base that a beginning of noise-induced sidebranching. In summary, there are at present no indications that the noise created by the walkers has a noticeable effect on the morphological evolution.

\section{CONCLUSIONS}

We have presented a new computational approach for multi-scale pattern formation in solidification. The method is efficient, robust, precise, easy to implement in both two and three dimensions, and parallelizable. Hence, it constitutes a powerful alternative to state of the art adaptive meshing and finite element techniques. We have illustrated its usefulness by simulating dendritic growth of a pure substance from its undercooled melt in an infinite geometry. Due to the fact that only a very limited amount of "geometry bookkeeping" is required, our method can be easily adapted to other experimental settings, such as directional solidification. In addition, the DMC algorithm is not limited to the present combination with the phase-field method, but can be used in conjunction with any method to solve the interface dynamics, as long as the diffusion equation is explicitly solved. The adaptation of our method to other diffusionlimited free boundary problems is straightforward; problems with several diffusion fields can be handled by introducing multiple species of walkers.
In view of the results presented here, there is a realistic prospect for direct simulations of solidification microstructures for experimentally relevant control parameters. An especially interesting prospect is to combine our method with a recently developed approach to quantitatively incorporate thermal fluctuations [31] in the phasefield model. Such an extension should make it possible to test noise-induced sidebranching theories 16,34 in three dimensions and for an undercooling range where detailed measurements of sidebranching characteristics are available [22, 26, 35, 36]. If thermal noise in the liquid region outside the buffer layer turns out to be unimportant for sidebranching, the straightforward addition of Langevin forces as in Ref. 31 in the finite-difference region (i.e. the buffer region plus the solid) should suffice for this extension. In contrast, if the noise from this region is important, a method to produce the correct level of noise in the walker region will need to be developed. Work concerning this issue is currently in progress.

To conclude, let us comment on some possible extensions and improvements of our method which will be necessary to address certain questions. Firstly, we have described the method here using an explicit integration scheme on the fine grid in the inner region, which enforces rather small time steps. We also tested an alternating direction Crank-Nicholson scheme in 2-d, which speeds up the calculations but makes it necessary to introduce corrective terms at the conversion boundary to guarantee the local heat conservation. Secondly, for the moment we use the stochastic algorithm only at the exterior of the dendrite; for other geometries, such as directional solidification where the volumes of solid and liquid are comparable, it might be useful to introduce a second stochastic region in the solid. It would also be desirable to combine our algorithm with more efficient memory managing techniques to overcome the limitations due to storage space. Finally, a completely open question is whether it is possible to combine our stochastic algorithm with a suitable method for simulating hydrodynamic equations. This would open the way for studies of the influence of convection on dendritic evolution at low undercooling, thereby extending in a non-trivial way recent studies that have been restricted to a relatively high undercooling regime 37.

\section{ACKNOWLEDGMENTS}

This research was supported by U.S. DOE Grant No. DE-FG02-92ER45471 and benefited from computer time at the National Energy Research Scientific Computing Center (NERSC) at Lawrence Berkeley National Laboratory and the Northeastern University Advanced Scientific Computation Center (NU-ASCC). We thank Youngyih Lee for providing the boundary integral results and Flavio Fenton for his help with the visualization. Fig. 8 was created using Advanced Visual Systems' AVS. 


\section{APPENDIX A: PHASE-FIELD METHOD}

We will briefly outline the main features of the phasefield method used for our simulations. More details can be found in Ref. 29.

The starting point is the free energy functional, Eq. (9), together with the equations of motion for the phase field and the temperature field, Eqs. (10) and (11). The free energy density in Eq. (9) is chosen to be of the form

$$
f(\psi, u)=-\frac{\psi^{2}}{2}+\frac{\psi^{4}}{4}+\lambda u \psi\left(1-2 \frac{\psi^{2}}{3}+\frac{\psi^{4}}{5}\right) .
$$

This function has the shape of a double well, with minima at $\psi= \pm 1$ corresponding to the solid and the liquid phases, respectively. Here, $u$ is the dimensionless temperature field, $\lambda$ is a dimensionless coupling constant, and the term proportional to $u$ on the RHS of Eq. (A1) "tilts" the double well in order to favor the solid (liquid) minimum when the temperature is below (above) the melting temperature. The coefficient $W(\hat{n})$ of the gradient term in the free energy (9) determines the thickness of the diffuse interface, i.e. the scale on which the phase field varies rapidly to connect the two equilibrium values. In addition, $W$ is related to the surface tension, and exploiting its dependence on the orientation of the interface allows to recover the anisotropic surface tension of Eq. (5) by choosing

$$
W(\hat{n})=W_{0} \frac{\gamma(\hat{n})}{\gamma_{0}} .
$$

The orientation $\hat{n}$ is defined in terms of the phase field by

$$
\hat{n}=\frac{\vec{\nabla} \psi}{|\vec{\nabla} \psi|}
$$

Note that this dependence of $W$ on $\psi$ has to be taken into account in performing the functional derivative, such that the explicit form of Eq. (10) becomes

$$
\begin{aligned}
\tau(\hat{n}) \partial_{t} \psi=[ & \left.\psi-\lambda u\left(1-\psi^{2}\right)\right]\left(1-\psi^{2}\right)+\vec{\nabla} \cdot\left[W(\hat{n})^{2} \vec{\nabla} \psi\right] \\
& +\partial_{x}\left(|\vec{\nabla} \psi|^{2} W(\hat{n}) \frac{\partial W(\hat{n})}{\partial\left(\partial_{x} \psi\right)}\right) \\
& +\partial_{y}\left(|\vec{\nabla} \psi|^{2} W(\hat{n}) \frac{\partial W(\hat{n})}{\partial\left(\partial_{y} \psi\right)}\right) \\
& +\partial_{z}\left(|\vec{\nabla} \psi|^{2} W(\hat{n}) \frac{\partial W(\hat{n})}{\partial\left(\partial_{z} \psi\right)}\right) .
\end{aligned}
$$

Next, we need to specify the orientation-dependent relaxation time $\tau(\hat{n})$ of the phase-field. In analogy with Eqs. (A2) and (5) we choose

$$
\tau(\hat{n})=\tau_{0}\left(1-3 \delta_{4}\right)\left[1+\frac{4 \delta_{4}}{1-3 \delta_{4}}\left(n_{x}^{4}+n_{y}^{4}+n_{z}^{4}\right)\right]
$$

where $\delta_{4}$ is the kinetic anisotropy.

The phase-field equations can be related to the original free boundary problem by the technique of matched asymptotic expansions. Details on this procedure can be found in Ref. [29]. As a result, we obtain expressions for the capillary length and the kinetic coefficient in terms of the phase-field parameters $W_{0}$ and $\tau(\hat{n})$ :

$$
\begin{gathered}
d_{0}=\frac{a_{1} W_{0}}{\lambda} \\
\beta(\hat{n})=\frac{a_{1}}{\lambda} \frac{\tau(\hat{n})}{W_{0}}\left(1-a_{2} \lambda \frac{W(\hat{n})^{2}}{D \tau(\hat{n})}\right),
\end{gathered}
$$

where $a_{1}=0.8839$ and $a_{2}=0.6267$ are numerical constants fixed by a solvability condition. There is an important difference between this result and earlier matched asymptotic expansions of the phase-field equations, due to a different choice of the expansion parameter. If the coupling constant $\lambda$ is used as the expansion parameter, the first order in $\lambda$ gives only the first term in Eq. (A7), while the complete expression is the result of an expansion to first order in the interface Péclet number, which is defined as the ratio of the interface thickness and a relevant macroscopic scale of the pattern (local radius of curvature or diffusion length). An important consequence of Eq. (A7) is that the kinetic coefficient and its anisotropy can be set to arbitrary values by a suitable choice of $\lambda$ and $\tau(\hat{n})$, and in particular we can achieve vanishing kinetics $(\beta(\hat{n})=0)$. Note that for a $\tau(\hat{n})$ as given by Eq. (A5), the kinetic coefficient cannot be made to vanish simultaneously in all directions, but for small anisotropies choosing $\delta_{4}=2 \epsilon_{4}$ is a sufficiently accurate approximation. Furthermore, the ratio $d_{0} / W_{0}$ can be decreased without changing the kinetics by simultaneously increasing $\lambda$ and the diffusivity $D$. This method dramatically increases the computational efficiency of the phase-field approach, because the interface width $W_{0}$ determines the grid spacing which must be used for an accurate numerical solution. For a physical system with fixed capillary length $d_{0}$, the number of floating point operations necessary to simulate dendritic evolution for some fixed time interval and system size scales $\sim\left(d_{0} / W_{0}\right)^{d+3}$ for the choice of phase-field parameters where the interface kinetics vanish (i.e. $D \tau / W_{0}^{2} \sim \lambda \sim W_{0} / d_{0}$ ), where $d$ is the spatial dimension [29].

We integrate the phase-field equations on a cubic grid with spacing $\Delta x$, All spatial derivatives are discretized using $(\Delta x)^{2}$-accurate finite difference formulas, and timestepping is performed by a standard Euler algorithm. The use of a regular grid induces small anisotropies in the surface tension and the kinetic coefficient. These effects have been precisely quantified in Ref. 29]. Since the grid has the same symmetry as the crystal we want to simulate, the presence of the lattice simply leads to small shifts in the surface tension anisotropy and in the kinetic parameters. For example, we obtain an effective surface tension anisotropy $\epsilon_{4}^{e}$ which 
is slightly smaller than the "bare" value $\epsilon_{4}$. Evidently, the use of this method restricts the simulation to crystals with symmetry axes aligned to the lattice, but this is not a severe limitation in the present study which focuses on the growth of single crystals.

[1] W. Kurz and D. J. Fisher, "Fundamentals of solidification", Trans Tech, Aedermannsdorf, Switzerland (1992).

[2] M. Matsushita, M. Sano, Y. Hayakawa, H. Honjo, and Y. Sawada, "Fractal structures of zinc metal leaves grown by electrodeposition", Phys. Rev. Lett. 53, 286 (1984).

[3] E. Ben-Jacob, H. Shmueli, O. Shochet, and A. Tenenbaum, "Adaptive self-organization during growth of bacterial colonies", Physica A 187, 378 (1992).

[4] R. J. Braun and M. T. Murray, J. Cryst. Growth 174, 41 (1997).

[5] A. Schmidt: "Computations of Three-Dimensional Dendrites with Finite Elements", J. Comp. Phys. 125, 293 (1996).

[6] N. Provatas, N. Goldenfeld, and J. Dantzig, "Efficient computation of dendritic microstructures using adaptive mesh refinement", Phys. Rev. Lett. 80, 3308 (1998), "Adaptive Mesh Refinement Computation of Solidification Microstructures Using Dynamic Data Structures", J. Comp. Phys. 148, 265 (1999).

[7] See for example S. E. Koonin and D. C. Meredith, "Computational Physics", Addison-Wesley, Redwood City (1992), chapter 8.

[8] L. P. Kadanoff, "Simulating Hydrodynamics: A Pedestrian Model", J. Stat. Phys. 39, 267 (1985).

[9] S. Liang, "Random-walk simulations of flow in Hele Shaw cells", Phys. Rev. A 33, 2663 (1986).

[10] T. Vicsek, "Pattern Formation in Diffusion-Limited Aggregation", Phys. Rev. Lett. 53, 2281 (1984).

[11] J. Nittmann and H. E. Stanley, "Tip splitting without interfacial tension and dendritic growth patterns arising from molecular anisotropy", Nature 321, 663 (1986).

[12] A. Karma, "Beyond Steady-State Lamellar Eutectic Growth", Phys. Rev. Lett. 59, 71 (1987).

[13] Y. Saito and T. Ueta, "Monte Carlo studies of equilibrium and growth shapes of a crystal", Phys. Rev. A 40, 3408 (1989)

[14] P. Meakin, "Diffusion-controlled cluster formation in 26-dimensional space", Phys. Rev. A 27, 1495 (1983).

[15] E. Brener, H. Müller-Krumbhaar, D. Temkin, "Structure formation and the morphology diagram of possible structures in two-dimensional diffusional growth", Phys. Rev. E 54, 2714 (1996).

[16] J. S. Langer, "Dendritic sidebranching in the threedimensional symmetric model in the presence of noise" Phys. Rev. A 36, 3350 (1987).

[17] M. Plapp and A. Karma, "Multiscale random-walk algorithm for simulating interfacial pattern formation", condmat/9906370

[18] A. Karma, Y. Lee, and M. Plapp, "Three-dimensional dendrite tip morphology at low undercooling", condmat/9909021

[19] J. S. Langer, "Instabilities and pattern formation in crystal growth", Rev. Mod. Phys. 52, 1 (1980).

[20] D. A. Kessler, J. Koplik, and H. Levine, "Pattern selection in fingered growth phenomena", Adv. Phys. 37, 255 (1988).

[21] E. A. Brener and V. I. Mel'nikov, "Pattern selection in two-dimensional dendritic growth", Adv. Phys. 40, 53 (1991).

[22] S.-C. Huang and M. E. Glicksman, "Fundamentals of Dendritic Solidification I. Steady-State Tip Growth", Acta Metall. 29, 701 (1981).

[23] E. R. Rubinstein and M. E.Glicksman, "Dendritic growth kinetics and structure I. Pivalic acid", J. Cryst. Growth 112, 84 (1991).

[24] M. Muschol, D. Liu, and H. Z. Cummins, "Surfacetension-anisotropy measurements of succinonitrile and pivalic acid: Comparison with microscopic solvability theory", Phys. Rev. A 46, 1038 (1992).

[25] M. E. Glicksman, M. B. Koss, and E. A. Winsa, "Dendritic Growth Velocities in Microgravity", Phys. Rev. Lett. 73, 573 (1994).

[26] U. Bisang and J. Bilgram, "Shape of the tip and the formation of sidebranches of xenon dendrites", Phys. Rev. E 54, 5309 (1996).

[27] J. A. Sethian and J. Strain, "Crystal Growth and Dendritic Solidification", J. Comp. Phys. 98, 231 (1992).

[28] T. Ihle and H. Müller-Krumbhaar, "Fractal and compact growth morphologies in phase transitions with diffusion transport", Phys. Rev. E 49, 2972 (1994).

[29] A. Karma and W.-J. Rappel, "Quantitative phase-field modeling of dendritic growth in two and three dimensions", Phys. Rev. E 57, 4323 (1998).

[30] For a brief historic overview and a comprehensive list of references concerning the phase-field method, see Ref. 29.

[31] A. Karma and W.-J. Rappel, "Phase-Field Model of Dendritic Sidebranching with Thermal Noise", condmat/9902017 (1999).

[32] Youngyih Lee, private communication.

[33] A. Barbieri and J. S. Langer, "Prediction of dendritic growth rates in the linearized solvability theory", Phys. Rev. A 39, 5314 (1989).

[34] E. Brener and D. Temkin, "Noise-induced sidebranching in the three-dimensional nonaxisymmetric dendritic growth", Phys. Rev. E 51, 351 (1995).

[35] A. Dougherty, P. D. Kaplan, and J. P. Gollub, "Development of Side Branching in Dendrite Crystal Growth", Phys. Rev. Lett. 58, 1652 (1987)

[36] Q. Li and C. Beckermann, "Scaling behavior of threedimensional dendrites", Phys. Rev. E 57, 3176 (1998)

[37] C. Beckermann, H.-J. Diepers, I. Steinbach, A. Karma, and X. Tong, "Modeling Melt Convection in Phase-Field Simulations of Solidification", J. Comp. Phys. 154, 468496 (1999). 